\title{
Dynamical Characteristics of Surface EMG Signals of Hand Grasps via Recurrence Plot
}

\author{
Gaoxiang Ouyang, Zhaojie Ju, Member, IEEE and Honghai Liu, Senior Member, IEEE
}

\begin{abstract}
Recognising human hand grasp movements through surface electromyogram (sEMG) is a challenging task. In this paper, we investigated non-linear measures based on recurrence plot, as a tool to evaluate the hidden dynamical characteristics of sEMG during four different hand movements. A series of experimental tests in this study show that the dynamical characteristics of SEMG data with recurrence quantification analysis (RQA) can distinguish different hand grasp movements. Meanwhile, adaptive neuro-fuzzy inference system (ANFIS) is applied to evaluate the performance of the above measures to identify the grasp movements. The experimental results shows that the recognition rate, $99.1 \%$, based on the combination of linear and non-linear measures is much higher than that with only linear measures, $93.4 \%$, or non-linear measures, $88.1 \%$. These results suggest that the RQA measures might be a potential tool to reveal the sEMG hidden characteristics of hand grasp movements and an effective supplement for the traditional linear grasp recognition methods.
\end{abstract}

Index Terms - Non-linear measures, surface electromyogram (sEMG), hand grasp, recurrence plot (RP)

\section{INTRODUCTION}

$\mathrm{T}$ HE surface electromyogram (sEMG) reflects both peripheral and central properties of the neuromuscular system and presents information of the neural activation of muscles [1]. Thus, the analysis of the sEMG signals is particularly attractive in that it provides relatively easy access to the physiological processes that allow the muscles to generate force and movement $[2,3]$. One important potential application using sEMG signals lies in controlling prosthetic devices $[4,5]$, for instance hand prostheses [6], due to the fact that sEMG signals contain the electrical activities of arm muscle contraction that undergo many complex transitions in different hand movements [7]. However, there are inherent difficulties in deriving a general model on the relationship between the recorded sEMG and hand gestures when humans perform contractions [8]. In order to control a prosthetic device, the fundamental challenge is to efficiently handle

Manuscript received November 8, 2012; revised February 24, 2013; accepted April 27, 2013. Date of publication xxxx xx, 2013; date of current version $\mathrm{xxxx} \mathrm{xx}, 2013$

Corresponding author: H. H. Liu (e-mail: honghai.liu@port.ac.uk). This work was supported by the Leverhulme Visiting Fellowship.

G. X. Ouyang, Z. J. Ju and H. Liu are with the Intelligent Systems \& Biomedical Robotics Group, School of Creative Technologies, University of Portsmouth, England, PO1 2DJ, UK (gx.ouyang@gmail.com; zhaojie.ju, honghai.liu@port.ac.uk).

Color versions of one or more of the figures in this paper are available online at http://ieeexplore.ieee.org.

Digital Object Identifier inserted by IEEE
sEMG signals and identify the intention of the users [9]. Meanwhile, the exploration of inherent features of sEMG signals could be of benefit in understanding the mechanisms of neuromuscular systems. Therefore, there is a growing interest in tackling this challenge [10].

Various methods have been proposed to extract valuable information from sEMG signals ranging from traditional linear methods such as time and frequency analysis to nonlinear methods. In the time domain, traditional methods of EMG amplitude analysis mainly consist of the mean absolute value, number of zero crossings, waveform length, and number of slope sign changes $[11,12]$. It should be noted that, during a sustained isometric contraction, there is a phenomenon called EMG spectrum compression. That is to say that there is an increase in the amplitude of the low frequency band and a relative decrease in the higher frequency band [13]. To some extent, these traditional methods of EMG amplitude have the capability of tracking muscular changes [12]. However, traditional methods of sEMG amplitude and spectral analysis are not effective in analysing complex transient signals [14]. In particular, the spectral analysis is based on an assumption that the observed variations of electrical field of muscle activity are time-invariant (stationary) processes [13]. Therefore, these traditional methods fail to detect the critical feature of sEMG data during transient human movement since this assumption is inconsistent with sEMG dynamics.

Nonlinear methods derived from the theory of nonlinear dynamical systems (also called chaos theory) recently have been proposed to analyse the sEMG signals [13]. These methods such as the Lyapunov exponents and fractal dimension extract an informative feature from SEMG data to detect the muscle status changes [15]. However, chaos-based approaches assume that the signal is stationary and originates from a low-dimensional, nonlinear system [16]. In practice, the sEMG signal is a non-stationary signal and stems from a highly nonlinear system, which is a complex signal embedded in noise [17]. It is thus promising to develop new methods to characterize sEMG changes in different neural activation of muscles based on nonlinear methods. Moreover, recently reported sEMG analysis techniques and tools have made it possible to extract the substantial meaningful information from recordings of the neuromuscular system $[10,13]$. Note that chaos-based approaches should be employed to analyse sEMG data in constrained conditions.

It is evident that nonlinear methods have significantly inspired research in sEMG signal analysis. For instance, a recurrence quantification analysis (RQA) was employed to analyse the non-linear dynamical characteristics of sEMG data 
[3]. One of its key features is the ability of describing nonlinear nature of short and non-stationary signal corrupted with noise [18]. RQA methods have been broadly applied to the analysis of physiological data, such as electroencephalogram data [19], fMRI data [20], heart signals [21], and EMG data $[22,23]$. A number of experimental studies showed that RQA methods have high potential of detecting changes in sEMG due to muscle contractions [24] or fatigue [25]. For example, Yuan et al. employed RQA measures and BP neural network to classify 8 forearm motions, which has been proved to achieve a desirable motion classification accuracy [26]. This study aims to investigate whether or not the RQA measures can extract the sEMG hidden characteristics of hand grasp movements and an effective supplement for the traditional linear grasp recognition methods.

\section{MATERIALS AND MethodS}

Eight ( 2 female, 6 male) healthy right-handed subjects volunteered for this study. Their ages range from 23 to 40 and average is 32.5 years; body height average is $175.5 \mathrm{~cm}$; body mass average is $70 \mathrm{~kg}$. All participants gave informed consent prior to the experiments according to the University of Portsmouth CCI Faculty Ethics Committee.

\section{A. Experimental Procedure}

The experiment consisted of both freely and different grasp gestures. Each type of grasps was repeated 10 times. Every motion lasted about 2 seconds. Between every two repetitions, participants had to relax hand for 2 seconds in the intermediate state, which is opening hand naturally without any muscle contraction. Once one motion with ten repetitions was finished, participants had to relax hand for 2 minutes before the next motion started. This was designed to overcome the effects of muscle fatigue.

\section{B. Data Collection}

The four EMG electrodes were applied to the subject's right forearm muscles, i.e. flexor carpi radialis (channel 1), flexor carpi ulnaris (channel 2), flexor pollicis longus (channel 3) and flexor digitorum profundus (channel 4). The sEMG data were recorded using DataLINK system from Biometrics LTD with a gel-skin contact area of about $4 \mathrm{~cm}^{2}$ for each bipolar electrode and a centre to centre recording distance of $20 \mathrm{~mm}$. The sampling frequency of DataLINK system in our experiment was set to be $1000 \mathrm{~Hz}$ and sEMG signals were amplified 1000 times and bandwidth is 20 to $460 \mathrm{~Hz}$ using a sEMG amplifier (SX230FW sEMG Amplifier, Biometrics LTD). To obtain good-quality signals, subjects were scrubbed with alcohol and shaved if necessary and then electrodes were applied over the body using the die cut medical grade doublesided adhesive tape. Electrodes locations were selected according to the Musculoskelet of these four muscles and confirmed by muscle specific contractions, which include manually resisted finger flexion, extension and abduction. The captured sEMG signals were visualized on a computer screen giving participants feedback to choose the positions of electrodes with stronger sEMG signals.
To investigate the dynamical characteristics of sEMG data during different grasp gestures, EMG signals were selected and dissected from no action (dataset I), grasp and lift an orange using five fingers (dataset II), grasp and lift a can full of rice using five fingers with the thumb abduction (dataset III) and hold and lift a dumbbell (dataset IV) intervals as shown in Fig.1. In this study, 80 4-channel 1-sec EMG epochs were selected for each dataset. Short (1-sec) EMG signals were used since the duration of the grasp gesture is only about a few seconds. The criteria for the selection of the EMG signals from hand grasps are that the interval is the first 1 second of hand hold and lift the objects.

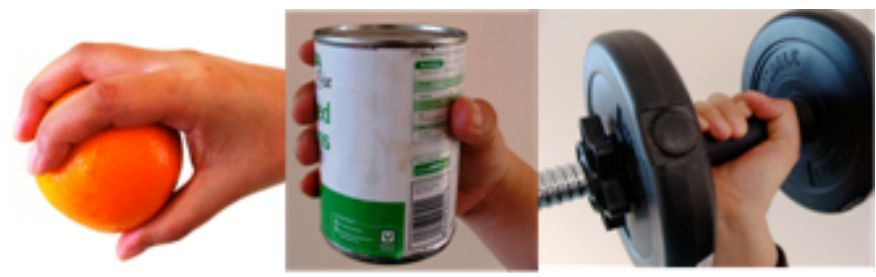

Fig. 1. Three hand grasping tasks.

\section{Phase Space Reconstruction}

EMG data is non-stationary time series. The first step in the analysis of a signal using non-linear dynamics theory is the reconstruction of the phase space trajectory of the signal [27]. The methods involves time delay are usually used to embed a scalar time series into a m-dimensional space, it is $x_{k}=\left(u_{k}, u_{k+\tau}, \cdots, u_{k+(m-1) \tau}\right)$, where $k=1,2, \cdots, L-(m-1) \tau$, $\tau$ is the delay time and $m$ is the embedding dimension, $m \geq 2$. The difficulty of this method is the choice of the delay time, $\tau$, and the minimum embedding dimension, $m$.

Takens' theorem assumes that the data is infinite and noisefree, where the delay time, $\tau$, can be chosen almost arbitrarily. However, real EMG data sets are always finite and noisy; the choice of the delay time must therefore be carefully considered. If $\tau$ is too small, the reconstructed vector is too close to serve as independent coordinates; if $\tau$ is too large, the reconstructed vector is independent and loses the connection with each other [28]. The most common method for choosing a proper time delay is based on detection of the first local minimum of the mutual information $(M I)$ function [29], since the first minimum of the $M I(\tau)$ portrays the time delay where the signal $u_{t+\tau}$ adds maximal information to the knowledge obtained from $u_{t}$ [29]. The $M I$ method produces non-linear characteristics of time series, so it is better to estimate time delay than linear autocorrelation functions.

As for the choice of the embedding dimension, if the $m$ is too small, the geometry is not entirely unfolded. As shown in Takens' embedding theorem, it certainly unfolds the attractor in $m$ dimensional space when $m<2 d+1$, where $d$ is the dimension of the attractor defined by the orbits. On the other hand, if $m$ is too large, it leads to excessive computations and enhances the problem of contamination by round off or instrumental error. Hence, it is crucial to choose a sufficiently large embedding dimension that contains the relevant 
dynamics in the presence of noise. There are a number of different criteria for choosing $m$ presented in the literature. Cao proposed a robust and efficient method that determines the minimum embedding dimension; it can overcome some shortcomings of false nearest neighbors [30]. In this study, the MI method [29] and the Cao's method [30] are employed to estimate the delay time and the embedding dimension, respectively.

\section{Recurrence Plot and RQA Measures}

Recurrence plots (RP), proposed by Eckmann et al. [31] describes the recurrence property of a deterministic dynamical system, i.e. visualizing the time dependent behavior of orbits $x_{i}$ in a phase space. The key step of RP is to calculate the following $N \times N$ matrix

$$
R_{i, j}(\varepsilon)=\Theta\left(\varepsilon-\left\|x_{i}-x_{j}\right\|\right), \quad i, j=1, \cdots, N
$$

where $N=L-(m-1) \tau, \varepsilon$ is a predefined cutoff distance, $\|\cdot\|$ is the norm (e.g. the Euclidean norm) and $\Theta(x)$ is a Heaviside function. The phase space vector $x_{i}$ can be reconstructed using Takens' time delay method, $x_{i}=\left(u_{i}, u_{i+\tau}, \cdots, u_{i+(m-1) \tau}\right)$ [27], based on the observations $u_{i}$. The cutoff distance $\varepsilon$ defines a sphere centered at $x_{j}$, if $x_{i}$ falls within this sphere, i.e. the state is close to $x_{j}$, then $R_{i, j}$ $=1$; otherwise $R_{i, j}=0$. The binary values of $R_{i, j}$ can be simply visualized with the colours black (1) and white (0). In short, RP can describe the characteristics of large-scale and smallscale patterns of a dynamical system [31].

In order to further investigate the properties of RP, several measures of complexity that quantify the small-scale structures in RP called recurrence quantification analysis (RQA), have been proposed. These measures are based on the recurrence point density and the diagonal line structures of the RP. For more details please refer to [18].

The classical measure of RQA is the recurrence rate $(R R)$

$$
R R(\varepsilon)=\frac{1}{N^{2}} \sum_{i, j=1}^{N} R_{i, j}(\varepsilon)
$$

which simply counts the black dots in the RP. $R R$ is a measure of the density of recurrence points.

The frequency distribution of the lengths $l$ of the diagonal structures in the RP is $P^{\varepsilon}(l)=\left\{l_{i} ; i=1,2, \cdots, N\right\}$. The ratio of recurrence points on the diagonal structures (of at least length $l_{\text {min }}$ ) to all recurrence points is called the DET, and is introduced as a determinism (or predictability) measure of the system. Its formulation is given as below:

$$
D E T=\sum_{l=l_{\min }}^{N} l P^{\varepsilon}(l) / \sum_{l=1}^{N} l P^{\varepsilon}(l)
$$

where $l_{\min }$ is the threshold, which excludes the diagonal lines formed by the tangential motion of a phase space trajectory, which in this study we fixed at $l_{\min }=2$.

ENTR refers to the Shannon entropy of the frequency distribution of the diagonal line lengths,

$$
\begin{aligned}
E N T R= & -\sum_{l=l_{\min }}^{N} p(l) \ln p(l) \\
& \text { where } p(l)=P(l) / \sum_{l=l_{\min }}^{N} P(l)
\end{aligned}
$$

ENTR is considered as a complexity measure of a deterministic structure in a dynamical system. The more complex the deterministic structure, the larger ENTR value.

A parameter specific to the RP is the cutoff distance $\varepsilon$. Several criteria for the choice of the cutoff distance $\varepsilon$ have been advocated in the literature [32]. One approach uses a fixed number of neighbours, $N_{n}$, for every point of the trajectory, called fixed amount of nearest neighbours (FAN) [18]). In this approach, the cutoff distance $\varepsilon_{i}$ changes for each state $x_{i}$ to ensure all columns of the RP have the same recurrence density. Using this neighbourhood criterion, $\varepsilon_{i}$ can be adjusted in such a way that the recurrence rate has a fixed predetermined value (i.e. $R R=N_{n} / N$ ) [18]; and the RP is invariant under enlarging or reducing of the amplitude of time series. In this paper, the DET and ENTR are computed for all 1 -sec EMG recordings (the number of data $L=1000$ ) with a fixed number of neighbours $N_{n}=50$. Using this $N_{n}$ value, the recurrence rate $R R$ is about 0.05 .

\section{E. Linear Analysis Methods}

In order to compare the extraction information of EMG between linear and nonlinear methods, six well known timedomain and frequency-domain linear methods, mean absolute value (MAV), number of zero crossings (ZC), waveform length (WL), number of slope sign changes (SS), mean frequency (MF) and peak frequency (PF) are also used to analyse the sEMG data. For more details, please refer to [11, 33].

\section{F. Adaptive Neuro-Fuzzy Inference System}

The ANFIS described by Jang Roger [34] is adopted to evaluate the ability and effectiveness of the above MPE measures in classifying the different seizure phases. The ANFIS learns features in the data set and adjusts the system parameters according to a given error criterion. It has been widely used in analysing the biological signals, such as EEG and EMG [35].

In order to improve the generalization, ANFIS classifiers are trained with the back propagation gradient descent method in combination with the least squares method. For each ANFIS classifier, nonlinear or/and linear features are used as inputs. The samples with target outputs, no action (dataset I), grasp an orange (dataset II), grasp a can (dataset III) and hold a dumbbell (dataset IV) are given the binary target values of $(0,0,0,1),(0,0,1,0),(0,1,0,0)$ and $(1,0,0,0)$, respectively.

\section{RESULtS}

\section{A. Choice of Time Delay and Emdedding Dimension}

The first step in RQA is the reconstruction of the $m$ dimensional phase space trajectory. The delay time $\tau$ and the 
embedding dimension $m$ of the EMG epochs are determined by using the $M I$ method and the Cao's method. Fig. 2A plots an original EMG epoch. Fig. 2B shows the mutual information versus the delay time of the EMG, the delay time $\tau$ at the first local minimum, $\tau=4$, is selected for the phase space reconstruction. Given the delay time, the Cao method is applied to determine the embedding dimension, the details can be found in [30]. As shown in Fig. 2C, after the $m=9, E 1$ does not change significantly over the dimension values, as a result we may take the minimum embedding dimension as 9 for this EMG epoch.
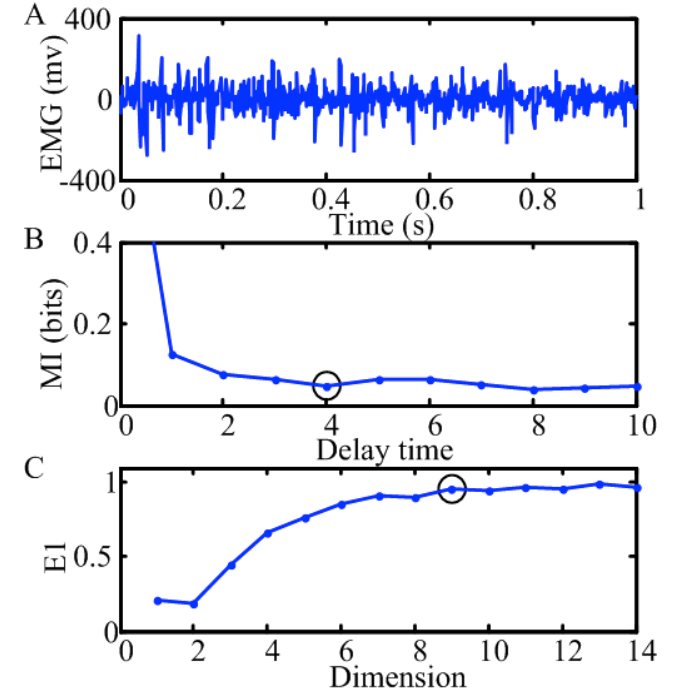

Fig. 2. The phase space reconstruction of a sEMG epochs. (A) an example for sEMG signal; (B) mutual information ( $M I$ ) versus delay time, the circle in (B) indicates the vicinity of $\tau$, which is at the first local minimum of $M I$, namely $\tau$ $=4$; (C) embedding dimension, the circle in (C) indicates $E 1$ stops significant changing at the $m=9$, so take the minimum embedding dimension as 9 .

Next, the MI method and Cao method are applied to all EMG epochs. The optimum values of $\tau$, based on detection of the first local minimum of the MI function, ranged from 2 to 8 samples (mean and standard deviation is $3.43 \pm 1.75$ ) for different EMG epochs. So the optimum delay time, $\tau=4$, is selected for the phase space reconstruction of the four EMG data sets. The optimum embedding dimension $m$ ranged from 6 to 12 (mean and standard deviation are $8.85 \pm 1.04$ ) for different EMG segments. Therefore, $m=9$ is suitable for the topologically proper reconstruction of the EMG data.

\section{B. RQA Measures of sEMG Data}

The RPs, as shown in Fig. 3, are constructed from the phase space trajectories of sEMG epochs. Fig. 3 is a representative example of sEMG signals from dataset IV, which recorded at flexor pollicis longus from subject 1. sEMG epoch and its RPs ( $m=9, \tau=4$ and $\left.N_{n}=50\right)$ are shown in Fig. 3. Visual inspections of RPs show that the diagonal lines structure in the $\mathrm{RP}$ of sEMG epoch is more regular than ones of dataset I and II sEMG epochs. In order to go beyond the visual impression yielded by RP, the RQA measures DET and ENTR are computed for sEMG epochs. In this example, DET and ENTR value of sEMG is 0.528 and 0.579 , respectively.

Next, the RQA measures, DET and ENTR, are applied to analyse all 12801 -sec sEMG epochs in this study $(80 \times 4-$ channel from each dataset I, II, III and IV). The averages of DET and ENTR of each sEMG wpochs are represented as a bar graph, as shown in Fig. 4(A) and (B), respectively. Fig. 4(A) shows the DET values of the no action are lower than those in the grasp and lift an orange, grasp and lift a can and hold and lift a dumbbell. For example, the DET values for the sEMG epochs from flexor digitorum profundus averaged $0.330 \pm 0.105,0.443 \pm 0.094,0.376 \pm 0.077$ and $0.563 \pm 0.110$ (mean $\pm \mathrm{SD}$ ) in dataset I, II III and IV, respectively. The same observation can also be mode for the ENTR in Fig. 4(B). These observations indicated that the alterations of muscle electrical activity dynamics could be characterized by the measures of RQA.

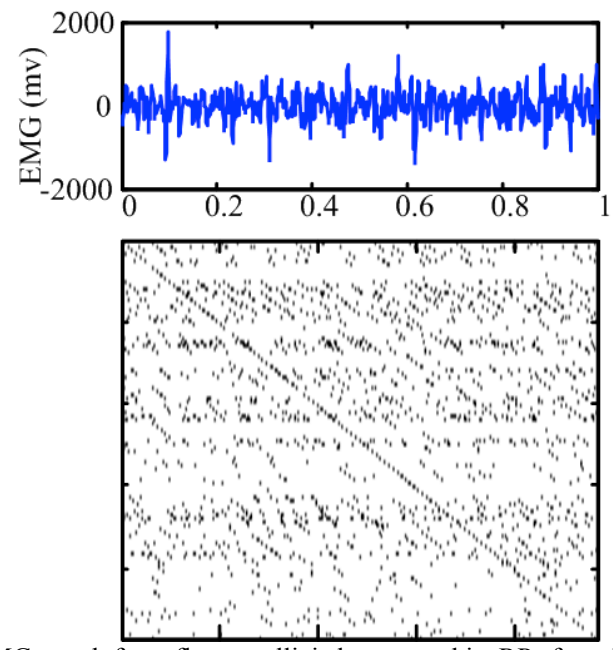

Fig. 3. sEMG epoch from flexor pollicis longus and its RPs from hold and lift a dumbbell (dataset IV).
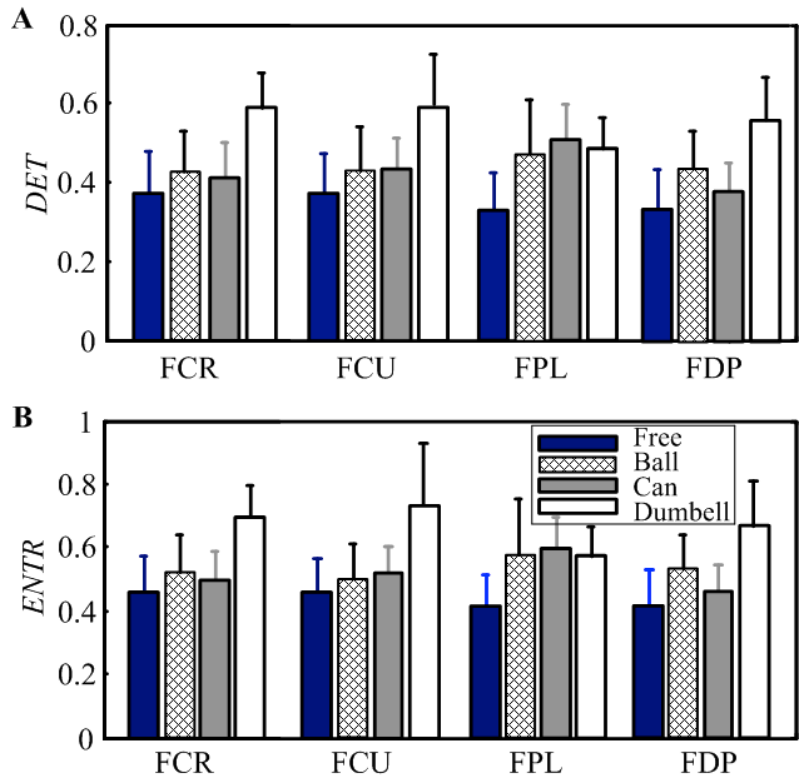

Fig. 4. Bar graph for the DET and ENTR values of the all sEMG epochs, grouped by no action (I), grasp and lift an orange (II) grasp and lift a can (III) and hold and lift a dumbbell (IV), from flexor carpi radialis (FCR), flexor carpi ulnaris (FCU), flexor pollicis longus (FPL) and flexor digitorum profundus (FDP), respectively. Height of each bar represents mean value, and the horizontal tick above each bar represents standard deviation. 
To test these observed mean differences statistically, the one-way ANOVA test is performed for DET and ENTR values of four different sets. As calculated, the critical value is $F_{\text {crit }}(3$, $316)=2.63$ at $\alpha=0.05$, at which point the test statistic must exceed to reject the test. In order to further investigate the EMG activities of different forearm muscles during hand grasp, statistical analysis of the DET and ENTR values in each dataset are carried out for flexor carpi radialis, flexor carpi ulnaris, flexor pollicis longus and flexor digitorum profundus, respectively. For example, the results of DET from flexor digitorum profundus are shown in Table I. It can be seen that the statistical test yields an $F$-test $(F=82.0)$ that is much higher than the threshold $F_{\text {crit }}(F=2.63)$. Thereby, this suggests the null hypothesis, i.e., no differences of $D E T$ between the four different groups from flexor digitorum profundus should be rejected. The similar results of statistical analysis can be obtained from other tests. The $F$ value is $81.1,80.5$ and 43.8 for $D E T$ from flexor carpi radialis, flexor carpi ulnaris and flexor pollicis longus, respectively. The $F$ value is 79.2, 82.0, 37.7 and 79.4 for ENTR from flexor carpi radialis, flexor carpi ulnaris, flexor pollicis longus and flexor digitorum profundus, respectively. The results suggest that the determinism of sEMG data in no action, grasp and lift an orange, grasp and lift a can and hold and lift a dumbbell are significantly different.

TABLE I

ONE-WAY ANOVA TEST

\begin{tabular}{lcccc}
\hline \hline $\begin{array}{l}\text { ANOVA } \\
\text { Source of } \\
\text { variation }\end{array}$ & $\begin{array}{c}\text { Sums of } \\
\text { squares } \\
\text { (SS) }\end{array}$ & $\begin{array}{c}\text { Degrees of } \\
\text { freedom } \\
\text { (DF) }\end{array}$ & $\begin{array}{c}\text { Mean } \\
\text { square } \\
\text { (MS) }\end{array}$ & $F$-test \\
\hline $\begin{array}{c}\text { Between } \\
\text { Samples }\end{array}$ & 2.46 & 3 & 0.820 & $82.0 \quad \boldsymbol{P}<0.05$ \\
$\begin{array}{c}\text { Within } \\
\text { Samples }\end{array}$ & 3.00 & 316 & 0.010 & \\
Total & 5.46 & 319 & & \\
\hline \hline
\end{tabular}

Thus, a Scheffe's post-hoc test (Matlab's multcompare function, statistics toolbox) [36] for all pairwise comparisons between the means is used to determine whether there is statistically significant difference between any two groups. For example, Table II shows the results of multiple comparisons analysis of DET values of sEMG data from flexor digitorum profundus. In this case, the threshold value for Scheffe's post-hoc test can be calculated as below:

$$
S_{t h}=\sqrt{\frac{4 * M S^{*} F_{c r i t}}{n}}=\sqrt{\frac{4 * 0.01 * 2.63}{80}}=0.036
$$

When the difference between treatment means is larger than the threshold value $S_{t h}$, there is a statistically significant difference between these two groups. The DET values for the sEMG epochs from flexor digitorum profundus averaged $0.330,0.443,0.376$ and 0.563 in dataset I, II, III and IV, respectively. The results suggest that the $D E T$ values for the sEMG data from flexor digitorum profundus during grasp an orange, grasp a can and hold a dumbbell each have significantly higher values than the no action, grasp an orange and grasp a can each have significantly lower values than hold a dumbbell, and grasp an orange significantly differs from grasp a can. Similar statistical results can be also obtained from the ENTR values. In short, it can be seen that the difference between different groups is statistically significant.

TABLE II

ONE-WAY PAIR-WISE COMPARISONS VIA SCHEFFE'S TEST

\begin{tabular}{cccc}
\hline \hline $\begin{array}{l}\text { Difference between } \\
\text { treatment means }\end{array}$ & II & III & IV \\
\hline I & $0.113^{*}$ & $0.046^{*}$ & $0.233^{*}$ \\
II & - & $0.067^{*}$ & $0.120^{*}$ \\
III & - & - & $0.187^{*}$ \\
\hline \hline * imply that the two treatment level means are statistically \\
different at the $a=0.05$ level $\left(S_{t h}=0.036\right)$.
\end{tabular}

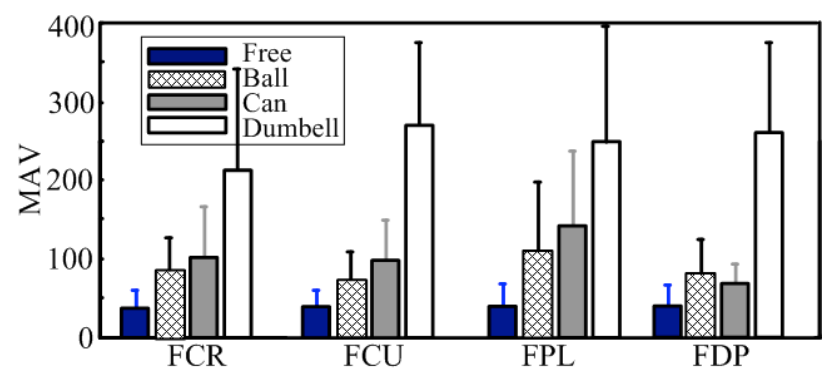

Fig. 5. Bar graph for the mean absolute values (MAV) of the all sEMG epochs, grouped by no action (I), grasp and lift an orange (II), grasp and lift a can (III) and hold and lift a dumbbell (IV), from flexor carpi radialis (FCR), flexor carpi ulnaris (FCU), flexor pollicis longus (FPL) and flexor digitorum profundus (FDP), respectively. Height of each bar represents mean value, and the horizontal tick above each bar represents standard deviation.

\section{Linear Analysis of sEMG Data}

The linear techniques mean absolute value (MAV), number of zero crossings (ZC), waveform length (WL), number of slope sign changes (SS), mean frequency (MF) and peak frequency (PF) are applied to analyse all 1280 1-sec sEMG epochs in this study $(80 \mathrm{x}$ 4-channel from each dataset I, II, III and IV). For example, the results of mean absolute value are shown in Fig. 5 and the results of one-way ANOVA test for $M A V$ from flexor digitorum profundus are shown in Table III. It can be seen that the statistical test yields an $F$-test $(F=172.4)$ that is much higher than the threshold $F_{\text {crit }}(F=2.63)$. Thereby, this suggests the null hypothesis, i.e., no differences of $M A V$ between the four different groups from flexor digitorum profundus should be rejected. The similar results of statistical analysis can be obtained from other tests. The $F$ value is 86.0 , 203.3 and 85.1 for $M A V$ from flexor carpi radialis, flexor carpi ulnaris and flexor pollicis longus, respectively. Table IV shows the results of multiple comparisons analysis of $M A V$ values of sEMG data from flexor digitorum profundus. The $M A V$ values for the sEMG epochs from flexor digitorum profundus averaged 39.5, 79.6, 67.7 and 255.6, respectively. It is found that the $M A V$ values for the sEMG data from flexor digitorum profundus during grasping an orange, grasping a can and holding a dumbbell each are significantly higher than that with no action. The same observation can also be made for the number of zero crossings, waveform length, number of slope sign changes, mean frequency and peak frequency. 
TABLE III

ONE-WAY ANOVA TEST

\begin{tabular}{ccccc}
\hline \multicolumn{5}{c}{ ONE-WAY ANOVA TEST } \\
$\begin{array}{l}\text { ANOurce } \\
\text { variation }\end{array}$ & $\begin{array}{c}\text { Sums of } \\
\text { squares } \\
\text { (SS) }\end{array}$ & $\begin{array}{c}\text { Degrees of } \\
\text { freedom } \\
\text { (DF) }\end{array}$ & $\begin{array}{c}\text { Mean } \\
\text { square } \\
\text { (MS) }\end{array}$ & $F$-test \\
\hline $\begin{array}{c}\text { Between } \\
\text { Samples } \\
\text { Within }\end{array}$ & 2309607 & 3 & 769869 & 172.4 \\
$\begin{array}{c}\text { Samples } \\
\text { Total }\end{array}$ & 1411307 & 316 & 4466 & \\
\hline \hline
\end{tabular}

TABLE IV

ONE-WAY PAIR-WISE COMPARISONS VIA SCHEFFE'S TEST

\begin{tabular}{cccc}
\hline \hline $\begin{array}{c}\text { Difference between } \\
\text { treatment means }\end{array}$ & II & III & IV \\
\hline I & $40.1^{*}$ & $28.2^{*}$ & $216.1^{*}$ \\
II & & 12.1 & $176.0^{*}$ \\
III & & & $187.9^{*}$ \\
\hline \hline
\end{tabular}

* imply that the two treatment level means are statistically different at the $a=0.05$ level $\left(S_{t h}=24.2\right)$.

\section{Classification}

The performance of the above measures to discriminate among groups is also evaluated by means of an adaptive neuro-fuzzy inference system (ANFIS) [34], and 10-fold cross-validations are employed to demonstrate the accuracy of classification [37]. First, the ability of the linear techniques in classifying different hand grasp is evaluated using the ANFIS. Four ANFIS classifiers are trained with the back-propagation gradient descent method in combination with the least squares method when the calculated six linear measures are used as input. Each of the ANFIS classifier is trained so that they are likely to be more accurate for one state of sEMG signals than the other states. Samples with target outputs sets I, II, III and IV are given the binary target values of $(0,0,0,1),(0,0,1,0)$, $(0,1,0,0)$ and $(1,0,0,0)$, respectively. Each ANFIS classifier is implemented by using the MATLAB software package (MATLAB version 7.0 with fuzzy logic toolbox). The classification results are illustrated in Table V. Of 320 hand movements in four groups, 299 are classified correctly. The total classification accuracy is $93.4 \%$.

TABLE V

CLASSIFICATION RESUlTS WITH LINEAR MEASURES

\begin{tabular}{ccccc}
\hline \hline \multirow{2}{*}{ Desired result } & $\begin{array}{c}\text { Output result } \\
\text { Free }\end{array}$ & Orange & Can & Dumbbell \\
\hline Free & 78 & 1 & 1 & 0 \\
Orange & 0 & 69 & 11 & 0 \\
Can & 0 & 6 & 74 & 0 \\
Dumbbell & 0 & 1 & 1 & 78 \\
\hline
\end{tabular}

Next, in order to investigate whether or not the $R Q A$ measures can effectively extract additional hidden information from sEMG data, the calculated two non-linear measures are used as input data in the ANFIS classifiers, and 10-fold crossvalidations are employed to demonstrate the performance of classification. The classification results are illustrated in Table VI. Of 320 hand movements in four groups, 282 are classified correctly. The total classification accuracy is $88.1 \%$.
TABLE VI

CLASSIFICATION RESUltS With RQA MEASURES

\begin{tabular}{ccccc}
\hline \hline Desired result & $\begin{array}{c}\text { Output result } \\
\text { Free }\end{array}$ & Orange & Can & Dumbbell \\
\hline Free & 61 & 1 & 18 & 0 \\
Orange & 0 & 78 & 2 & 0 \\
Can & 11 & 6 & 63 & 0 \\
Dumbbell & 0 & 0 & 0 & 80 \\
\hline
\end{tabular}

Finally, the all calculated linear and non-linear measures are used as input data in the ANFIS classifier. Classification results of the ANFIS model revealed that, as listed in Table VII, only 2 sEMG segments from grasping a can are misclassified by ANFIS as grasping an orange sEMG segments and 1 sEMG segment from grasping an orange is mistaken as grasping a can. The total classification accuracy of ANFIS model is $99.1 \%$ by using 10 -fold cross-validations.

TABLE VII

CLASSIFICATION RESULTS WITH LINEAR AND RQA MEASURES

\begin{tabular}{ccccc}
\hline \hline Desired result & $\begin{array}{c}\text { Output result } \\
\text { Free }\end{array}$ & Orange & Can & Dumbbell \\
\hline Free & 80 & 0 & 0 & 0 \\
Orange & 0 & 79 & 1 & 0 \\
Can & 0 & 2 & 78 & 0 \\
Dumbbell & 0 & 0 & 0 & 80 \\
\hline
\end{tabular}

\section{DISCUSSIONS}

The sEMG signal is a measure of the summed activity of a number of motor unit action potentials (MUAP) lying in the vicinity of the recording electrode, and it may provide insight into the neural activation and dynamics of muscles [38, 39]. The composition of a sEMG signal from MUAPs results in a nonlinear and stochastic signal because of the different firing rates and the large number of motor units that contribute [40]. Thus, the sEMG signals are complex data that needs to be condensed with useful information; analysis of sEMG using nonlinear dynamics can provide a better insight into the complex relation between sEMG and muscle activities. In particular, nonlinear sEMG analysis has opened up a range of new perspectives for the study of normal and disturbed neuromuscular function [25] and its development to a new interdisciplinary field of nonlinear muscle dynamics [41]. Therefore, exploration of hidden dynamical structures within sEMG signals is of both basic and clinical interest and has attracted more and more attention [42, 43].

In this study, we have analysed non-linear dynamical characteristics in sEMG data during different hand grasp states using the RQA measure. It is found that there is a significant increase of the determinism of the sEMG data from no action state to hand grasp states. Similar results have been reported previously. In [44], the RQA method was applied to indicate the deterministic dynamics of the $\mathrm{SEMG}$ signals during the muscle state changes. In [3], the measure DET is used to 
detect the hidden rhythms in sEMG signals; the high values of $D E T$ in the sEMG signals during the contraction are found, and it was shown that DET is more effective than median frequency in detecting sEMG changes determined by brisk transients of force output. The increase in determinism is classically attributed to sEMG self-organization into more periodic waves, this process which may be caused by an increase in the probability that motor units will discharge in unity [25].

Furthermore, our results showed that there is a significant difference in the determinism of the sEMG data from forearm muscles among grasp an orange, grasp a can and hold a dumbbell. On one hand, our results demonstrated that the $D E T$ values for the sEMG data from flexor digitorum profundus have significantly higher values during hold a dumbbell than during grasp an orange and a can. This finding is consistent with the fact that the contractions of flexor digitorum profundus during hold a dumbbell are stronger than those during grasp an orange and a can. In terms of anatomy, it is well known that the flexor digitorum profundus muscle is a muscle of the human forearm that acts to flex the fingers [45]. Then, the ANFIS is applied to evaluate the performance of non-linear measures to discriminate among four hand grasp movements. A total classification accuracy of $88.1 \%$ is achieved. Moreover, the hand gestures of grasp a can and hold a dumbbell can be separated nicely when using the DET and ENTR measures.

Finally, the classical linear techniques are also used to extract the features from sEMG data. It is found that the high mean absolute values in the sEMG signals during the contraction are found. These results are consistent with the previous works that muscles in the process of contraction exhibit the increases in sEMG amplitude [11]. Then, the ANFIS is applied to evaluate the performance of linear measures to discriminate among four hand grasp movements. A total classification accuracy of $93.4 \%$ is achieved based on the traditional linear techniques. Moreover, it is found that the linear measures can provide a better separability between no action and grasp an object than the non-linear measures. Additionally, a total classification accuracy of $99.1 \%$ is achieved based on the combination of linear and non-linear measures. Thus, these results suggest that both linear and nonlinear measures of sEMG may contribute to the understanding of the electrical activities of muscle contraction during hand grasp, and our findings provide further support for the notion that different information about muscle electrical activities may be extracted using linear and non-linear measures. Another significant note is that the RQA measures of a single channel sEMG of $1 \mathrm{~s}$ could be calculated in less than $20 \mathrm{~ms}$ by using MATLAB (Math Works Inc.) on a $2.4 \mathrm{GHz}$ personal computer in this study. Therefore the combination of RQA measures and linear measures in the sEMG data has the potential of providing the basis for designing a real-time hand grasp movement detection system.

It is challenging that employing sEMG, due to its highly dynamic nature, to detect the changes of muscles for musculoskeletal assessment and prosthetic control. It is expected that the reported findings would lead to a solution for sEMG in-depth understanding and further reduction of patients' conscious efforts in monitoring dynamic hand motion of controlled prosthetic devices. One of the future works is targeted to investigate non-linear classifiers to further improve the classification performance for more natural hand motions $[46,47]$.

\section{REFERENCES}

[1] D. Farina, R. Merletti, and R. M. Enoka, "The extraction of neural strategies from the surface EMG," J Appl Physiol, vol. 96, pp. 1486-95, Apr 2004.

[2] H. Ghasemzadeh, R. Jafari, and B. Prabhakaran, "A body sensor network with electromyogram and inertial sensors: multimodal interpretation of muscular activities," IEEE Trans Inf Technol Biomed, vol. 14, pp. 198-206, Mar 2010.

[3] G. Filligoi and F. Felici, "Detection of hidden rhythms in surface EMG signals with a non-linear time-series tool," Med Eng Phys, vol. 21, pp. 439-48, Jul-Sep 1999.

[4] P. K. Artemiadis and K. J. Kyriakopoulos, "An EMG-based robot control scheme robust to time-varying EMG signal features," IEEE Trans Inf Technol Biomed, vol. 14, pp. 582-8, May 2010.

[5] Y. H. Yin, Y. J. Fan, and L. D. Xu, "EMG and EPP-integrated human-machine interface between the paralyzed and rehabilitation exoskeleton," IEEE Trans Inf Technol Biomed, vol. 16, pp. 542-9, Jul 2012.

[6] C. Cipriani, C. Antfolk, C. Balkenius, B. Rosen, G. Lundborg, M. C. Carrozza, et al., "A novel concept for a prosthetic hand with a bidirectional interface: a feasibility study," IEEE Trans Biomed Eng, vol. 56, pp. 2739-43, Nov 2009.

[7] F. V. Tenore, A. Ramos, A. Fahmy, S. Acharya, R. EtienneCummings, and N. V. Thakor, "Decoding of individuated finger movements using surface electromyography," IEEE Trans Biomed Eng, vol. 56, pp. 1427-34, May 2009.

[8] H. Liu, "Exploring human hand capabilities into embedded multifingered object manipulation," IEEE Transactions on Industrial Informatics, vol. 7, pp. 389-398, 2011.

[9] S. Kwon and J. Kim, "Real-time upper limb motion estimation from surface electromyography and joint angular velocities using an artificial neural network for human-machine cooperation," IEEE Trans Inf Technol Biomed, vol. 15, pp. 522-30, Jul 2011.

[10] R. Merletti, M. Aventaggiato, A. Botter, A. Holobar, H. Marateb, and T. M. Vieira, "Advances in surface EMG: recent progress in detection and processing techniques," Crit Rev Biomed Eng, vol. 38, pp. 305-45, 2010.

[11] B. Hudgins, P. Parker, and R. N. Scott, "A new strategy for multifunction myoelectric control," IEEE Trans Biomed Eng, vol. 40, pp. 82-94, Jan 1993.

[12] G. Li, A. E. Schultz, and T. A. Kuiken, "Quantifying pattern recognition-based myoelectric control of multifunctional transradial prostheses," IEEE Trans Neural Syst Rehabil Eng, vol. 18, pp. 185-92, Apr 2010.

[13] H. B. Xie, J. Y. Guo, and Y. P. Zheng, "Fuzzy approximate entropy analysis of chaotic and natural complex systems: detecting muscle fatigue using electromyography signals," Ann Biomed Eng, vol. 38, pp. 1483-96, Apr 2010.

[14] J. Duchene and F. Goubel, "Surface electromyogram during voluntary contraction: processing tools and relation to physiological events," Crit Rev Biomed Eng, vol. 21, pp. 313-97, 1993.

[15] X. Hu, Z. Z. Wang, and X. M. Ren, "Classification of surface EMG signal with fractal dimension," J Zhejiang Univ Sci B, vol. 6, pp. 844-8, Aug 2005.

[16] J. P. Eckmann and D. Ruelle, "FUNDAMENTAL LIMITATIONS FOR ESTIMATING DIMENSIONS AND LYAPUNOV EXPONENTS IN DYNAMIC-SYSTEMS," Physica D, vol. 56, pp. 185-187, May 1992.

[17] M. Akay, "Nonlinear biomedical signal processing," in Dynamic Analysis and Modeling. vol. II, ed: IEEE Press, 2001. 
[18] N. Marwan, M. C. Romano, M. Thiel, and J. Kurths, "Recurrence plots for the analysis of complex systems," Physics ReportsReview Section of Physics Letters, vol. 438, pp. 237-329, Jan 2007.

[19] G. Ouyang, X. Li, C. Dang, and D. A. Richards, "Using recurrence plot for determinism analysis of EEG recordings in genetic absence epilepsy rats," Clin Neurophysiol, vol. 119, pp. 1747-55, Aug 2008.

[20] M. Bianciardi, P. Sirabella, G. E. Hagberg, A. Giuliani, J. P. Zbilut, and A. Colosimo, "Model-free analysis of brain fMRI data by recurrence quantification," Neuroimage, vol. 37, pp. 489-503, Aug 152007.

[21] H. Yang, "Multiscale recurrence quantification analysis of spatial cardiac vectorcardiogram signals," IEEE Trans Biomed Eng, vol. 58, pp. 339-47, Feb 2011.

[22] A. Schmied and M. Descarreaux, "Reliability of EMG determinism to detect changes in motor unit synchrony and coherence during submaximal contraction," J Neurosci Methods, vol. 196, pp. 23846, Mar 302011.

[23] J. L. Dideriksen, D. Falla, M. Baekgaard, M. L. Mogensen, K. L. Steimle, and D. Farina, "Comparison between the degree of motor unit short-term synchronization and recurrence quantification analysis of the surface EMG in two human muscles," Clin Neurophysiol, vol. 120, pp. 2086-92, Dec 2009.

[24] S. M. Rissanen, M. Kankaanpaa, M. P. Tarvainen, A. Y. Meigal, J. Nuutinen, I. M. Tarkka, et al., "Analysis of dynamic voluntary muscle contractions in Parkinson's disease," IEEE Trans Biomed Eng, vol. 56, pp. 2280-8, Sep 2009.

[25] C. Morana, S. Ramdani, S. Perrey, and A. Varray, "Recurrence quantification analysis of surface electromyographic signal: sensitivity to potentiation and neuromuscular fatigue," $J$ Neurosci Methods, vol. 177, pp. 73-9, Feb 152009.

[26] C. Yuan, X. Zhu, G. Liu, M. Lei, and Ieee, "Classification of the Surface EMG Signal Using RQA Based Representations," in 2008 Ieee International Joint Conference on Neural Networks, Vols 1-8, ed, 2008, pp. 2106-2111.

[27] N. H. Packard, J. P. Crutchfield, J. D. Farmer, and R. S. Shaw, "GEOMETRY FROM A TIME-SERIES," Physical Review Letters, vol. 45, pp. 712-716, 1980.

[28] H. S. Kim, R. Eykholt, and J. D. Salas, "Nonlinear dynamics, delay times, and embedding windows," Physica D, vol. 127, pp. 48-60, Mar 1999.

[29] A. M. Fraser and H. L. Swinney, "INDEPENDENT COORDINATES FOR STRANGE ATTRACTORS FROM MUTUAL INFORMATION," Physical Review A, vol. 33, pp. 1134-1140, Feb 1986.

[30] L. Y. Cao, "Practical method for determining the minimum embedding dimension of a scalar time series," Physica D, vol. 110, pp. 43-50, Dec 11997.

[31] J. P. Eckmann, S. O. Kamphorst, and D. Ruelle, "RECURRENCE PLOTS OF DYNAMIC-SYSTEMS," Europhysics Letters, vol. 4, pp. 973-977, Nov 1987.

[32] L. Matassini, H. Kantz, J. Holyst, and R. Hegger, "Optimizing of recurrence plots for noise reduction," Phys Rev E Stat Nonlin Soft Matter Phys, vol. 65, p. 021102, Feb 2002.

[33] G. Fele-Zorz, G. Kavsek, Z. Novak-Antolic, and F. Jager, "A comparison of various linear and non-linear signal processing techniques to separate uterine EMG records of term and pre-term delivery groups," Med. Biol. Eng. Comput., vol. 46, pp. 911-22, 2008.

[34] J. S. R. Jang, "ANFIS - ADAPTIVE-NETWORK-BASED FUZZY INFERENCE SYSTEM," Ieee Transactions on Systems Man and Cybernetics, vol. 23, pp. 665-685, May-Jun 1993.

[35] R. T. Lauer, B. T. Smith, and R. R. Betz, "Application of a neurofuzzy network for gait event detection using electromyography in the child with cerebral palsy," IEEE Trans Biomed Eng, vol. 52, pp. 1532-40, Sep 2005

[36] R. V. Hogg and J. Ledolter, Engineering Statistics: MacMillan Publishing Company, 1987.

[37] C. D. Katsis, N. Katertsidis, G. Ganiatsas, and D. I. Fotiadis, "Toward emotion recognition in car-racing drivers: A biosignal processing approach," Ieee Transactions on Systems Man and Cybernetics Part a-Systems and Humans, vol. 38, pp. 502-512, May 2008.

[38] X. Li, Y. C. Wang, N. L. Suresh, W. Z. Rymer, and P. Zhou, "Motor unit number reductions in paretic muscles of stroke survivors," IEEE Trans Inf Technol Biomed, vol. 15, pp. 505-12, Jul 2011.

[39] J. S. Karlsson, K. Roeleveld, C. Gronlund, A. Holtermann, and N. Ostlund, "Signal processing of the surface electromyogram to gain insight into neuromuscular physiology," Philos Transact A Math Phys Eng Sci, vol. 367, pp. 337-56, Jan 282009.

[40] F. D. Farfan, J. C. Politti, and C. J. Felice, "Evaluation of EMG processing techniques using Information Theory," Biomed Eng Online, vol. 9, p. 72, 2010.

[41] H. Geyer and H. Herr, "A muscle-reflex model that encodes principles of legged mechanics produces human walking dynamics and muscle activities," IEEE Trans Neural Syst Rehabil Eng, vol. 18, pp. 263-73, Jun 2010.

[42] P. Palmes, W. T. Ang, F. Widjaja, L. C. Tan, and W. L. Au, "Pattern mining of multichannel sEMG for tremor classification," IEEE Trans Biomed Eng, vol. 57, pp. 2795-805, Dec 2010.

[43] D. Rodrick and W. Karwowski, "Nonlinear dynamical behavior of surface electromyographical signals of biceps muscle under two simulated static work postures," Nonlinear Dynamics Psychol Life Sci, vol. 10, pp. 21-35, Jan 2006.

[44] C. L. Webber, Jr., M. A. Schmidt, and J. M. Walsh, "Influence of isometric loading on biceps EMG dynamics as assessed by linear and nonlinear tools," J Appl Physiol, vol. 78, pp. 814-22, Mar 1995. G. R. Naik, D. K. Kumar, and Jayadeva, "Twin SVM for gesture classification using the surface electromyogram," IEEE Trans Inf Technol Biomed, vol. 14, pp. 301-8, Mar 2010.

[46] Ju, Z. and Liu, H. "Fuzzy Gaussian Mixture Models, Pattern Recognition", 45(3): 1146-1158, 2012

[47] Ju, Z. and Liu, H. "Human Hand Motion Analysis with Multisensory Information", IEEE/ASME Transactions on Mechatronics , vol. PP, no.99, pp.1,11, 0, doi: 10.1109/TMECH.2013.2240312

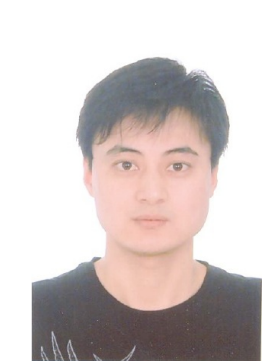

Gaoxiang Ouyang received the B.S. in automation and the M.S. in control theory and control engineering both from Yanshan University, China, in 2002 and 2004 respectively, and the Ph.D. degree at the Department of Manufacturing Engineering, City University of Hong Kong in 2010.

Dr Ouyang is a Lerverhulme Visting Fellow in the intelligent systems and biomedical robotics group at the University of Portsmouth. His research interests are bio-signal analysis, neural engineering, and dynamics system. He has published over twenty refereed international journal and conference papers.

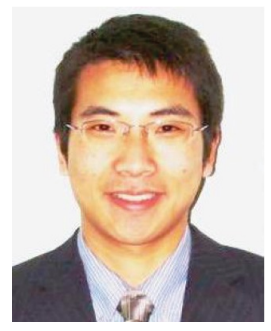

Zhaojie Ju (M'08) received the B.S. in automatic control and the M.S. in intelligent robotics both from Huazhong University of Science and Technology, China, in 2005 and 2007 respectively, and the Ph.D. degree in intelligent robotics at the University of Portsmouth, UK, in 2010.

$\mathrm{Dr} \mathrm{Ju}$ is currently a Lecturer in the School of Creative Technologies, University of Portsmouth, UK. He previously held research appointments in the Department of Computer Science, University College London and Intelligent Systems and Biomedical Robotics group, University of Portsmouth, UK. His research interests are in machine intelligence, robot learning, pattern recognition and their applications in robotic/prosthetic hand control and human-robot interaction.

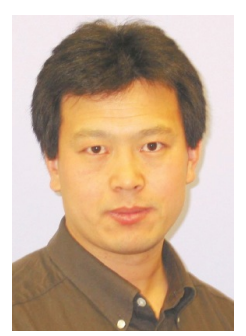

Honghai Liu (M'02-SM'06) received his Ph.D degree in robotics from King's college London, UK, in 2003.

$\mathrm{He}$ is currently a Professor of intelligent systems with the University of Portsmouth, Portsmouth, UK. He previously held research appointments at the Universities of London and Aberdeen, and project leader appointments in large-scale industrial control and system integration industry. $\mathrm{He}$ is interested in biomechatronics, pattern recognition, intelligent video analytics, intelligent robotics and their practical applications with an emphasis on approaches that could make contribution to the intelligent connection of perception to action using 
contextual information. He is Associate Editor of IEEE Transactions on Industrial Informatics, IEEE Transactions on Fuzzy Systems, IEEE

Transactions on Human-Machine Systems.

Dr Liu is a Fellow of the Institution of Engineering and Technology. 